\title{
A study to compare the effects of aerobic exercises and yoga on depression and maternal anxiety orienting among primiparous women
}

\author{
G. Yuvarani ${ }^{1}$, R. Manoranjitham ${ }^{2}$, G. Tharani ${ }^{3}$, K. Kamatchi ${ }^{4}$, Vaishnavi G. ${ }^{5}$ \\ ${ }^{1}$ Associate Professor, ${ }^{2}$ Postgraduate, ${ }^{3,4,5}$ Assistant Professor, Faculty of Physiotherapy, Dr. M.G.R. Educational and Research \\ Institute, Chennai 77, Tamil Nadu, India
}

(Received: March 2020 Revised: July $2020 \quad$ Accepted: August 2020)

Corresponding author: G. Yuvarani. Email: yuvarani.physio@drmrgdu.ac.in

\begin{abstract}
Introduction and Aim: Primiparous women undergo stages of depression or anxiety during pregnancy especially the risk of preterm birth, breast-feeding problems and disruption of the mother-infant attachment. It is a most common psychiatric disorder and is associated with a greater risk of low fetal quality, Pregnancy complication and developmental disorder of the child. During pregnancy 10-15\% women were affected with depression and the incidence increases in low economic status. It is associated with greater risk of prenatal and perinatal complication. In recent years the physical therapy is associated with psychological health in pregnant women. Physical activity during pregnancy is more beneficial to the maternal fetal unit and it may prevent recurrence of maternal disorders. The objective of this study is to compare the effects of aerobic exercise and yoga on depression and maternal anxiety orienting among primiparous women.
\end{abstract}

Materials and Methods: This experimental study was conducted with 30 subjects at ACS Medical College and Hospital, from January 2018 to March 2018. The outcome measures of CES-D scale were used to assess the severity of depression and anxiety. Group A received Aerobic exercise for 30 minutes initially started with warm up for 10 minutes and followed by 10 minutes stretching and 10 minutes relaxation. Group B received yoga for 20 minutes up to 3 months. The treatment duration was followed from the 16 to 20 weeks of gestation for 3 months.

Results: On comparing the mean values of group A and group B on centre for epidemiological studies depression scale score, it showed significant decrease in the post test mean values of group A (24.60) and group B (24.40) at $\mathrm{P} \leq 0.001$. Hence both the groups were equally effective in decreasing the depression and maternal anxiety among primiparous women.

Conclusion: This study concluded that the aerobic and yoga showed significant effect in both groups for reducing the symptoms of depression and anxiety among primiparous women.

Keywords: Primiparous women; aerobic exercises; yoga; CES - D scale.

\section{INTRODUCTION}

$\mathrm{P}$ regnancy is a state of carrying a developing embryo or fetus within the female body and it usually lasts for 40 weeks from the last menstrual cycle. Pregnancy is a time of increasing vulnerability for the development of anxiety and depression are the most common mental problem during pregnancy (1). It occurs more frequently during second and third trimester of pregnancy than the first trimester. The prevalence of antenatal was found to be ranging from 9.18\%-65\% northern, southern and western part of the India. Several risk factors predispose to depression during pregnancy. The major factor behind the complication are poor antenatal care, poor nutrition and lack of education (2). Primiparous defined as woman who is pregnant for first time. Primiparous defined as woman who is pregnant for first time. Prenatal depression is one of the leading cause that affects the physical and mental health of the mother and also the infant.. For example, children born to depressed mothers suffer from low birth weights, elevated resting heart rates, developmental delays and prematurity, increased physiological reactivity, and behavior problems in childhood and adolescence than children of nondepressed mothers (3).

Prenatal anxiety effect on pregnancy include increased cortisol level, pro-inflammatory and cytokine are effect on the neonatal include lower gestational age, less breast feeding (4). Depression and anxiety occurs due to high level of cortisol, secreted by the action of sympathetic nervous system. Further the cortisol has the ability to cross the placenta and affect the fetal growth (5). Pharmacological treatment such as Citalopram and Zoloft are anti-anxiety and anti-depressant drugs are proven to be effective in reducing the severity of depression and anxiety but has side effect such as birth defects, premature growth, constipation, nausea ,vomiting, dry mouth, drowsiness, dizziness etc., Non-pharmacological treatment specifically aerobic exercise and yoga are effective in treating depression and anxiety problems during pregnancy. Aerobic exercise (low-step level, walking, and stationary bicycling) is an accessible and convenient method that is proven to reduce the severity of depression and anxiety (6). It was practiced for 12 weeks (3 months) for 60 minutes, 3 days per week. It increases 
the temperature of the body and, it produces relaxation. Further, it stimulates the b-endorphins which produces positive feelings and promoting an active lifestyle. Yoga is an effective method to treat depression and anxiety. Yoga may produce improvement in stress levels, quality of life. It reduces the depression and anxiety by activating the parasympathetic nerves system, it acts against the sympathetic nervous system, and reduces the level of cortisol. Asana and pranayama is useful to reduce the depression and anxiety, asana is a body posture and pranayama is breathing exercise (7). Pranayama is most useful and effective in reducing depression and anxiety in primiparous woman. Centre for epidemiological studies dépression scale (CES-D), consists of 20 questions, score ranging between 0-60, higher the score more is the level of depression and anxiety (8). However, there is not sufficient research done to find out the effect of aerobic exercise and yoga on depression and anxiety on primiparous women. Hence, this study is intended to compare the effects of Aerobic exercise and yoga on primiparous women with depression and anxiety.

\section{MATERIALS AND METHODS}

This experimental study was conducted with 30 subjects at ACS Medical College and Hospital, from January 2018 to March 2018. The outcome measures of CES-D scale were used to assess the severity of depression and anxiety. Group A received aerobic exercise for 30 minutes initially started with warm up for 10 minutes and followed by 10 minutes stretching and 10 minutes relaxation. Group B received yoga for 20 minutes upto 3 months. The treatment duration was followed from the 16 to 20 weeks of gestation for 3 months.

\section{Background of the study}

The need of this study is that nowadays pregnant women especially primiparous pregnant women are suffering with depression and maternal anxiety. It is a most common psychiatric disorder and is associated with a greater risk of low fetal quality, Pregnancy complication and developmental disorder of the child. In India the prevalence range for primiparous women with anxiety was $56.5 \%$ and depression was $14.8 \%$. Depression is a disorder which is a general health concern throughout the world with prevalence around $10.4 \%$ among pregnant women. During pregnancy $10-15 \%$ women were affected with depression and the incidence increases in low economic status. It is associated with greater risk of prenatal and perinatal complications. Prenatal intervention for depressed pregnant women is physical therapy, antidepressants, psychotherapy, yoga, Aerobic exercise, supervised exercise etc., In recent years the physical therapy is associated with psychological health in pregnant women. Physical activity during pregnancy is more beneficial to the maternal fetal unit and it may prevent recurrence of maternal disorders.

Precautions for exercise: Drink plenty of water before and after the exercise, finish eating at least one hour before the exercise, avoid potholes, and rock other obstacle areas. Get up slowly and gradually to prevent dizziness, proper shoes should be used to exercise and stop exercising if any giddiness, chest, pelvic pains occur.

\section{Low-step level}

The patients were made to stand in front of the 4 inches step stool, and they were asked to place one leg on stool and slowly place the other leg also, and then slowly they brought their legs back to the starting position. This exercise was practiced for 10 minutes daily.

Walking: The patients were asked to stand keeping their back straight. They were asked to place their head and chin in correct level. Make the patient to swing their arms during walking for good balance.

Stationary bicycling: The patients were asked to sit on bicycle and placing their foot on the pedals. Legs were kept straight with knees bent at a 90 degree, and they were asked to gently press their one side cycle pedal. They were asked to practice for 10 minutes, for 5 days in a week.

Group B received Yoga for 20 minutes. One session per week for 3 months. Yoga was an effective method for prenatally depressed women by increasing the vagal activity. They were instructed to practice only basic sitting, kneeling standing yoga poses such as, Utkatasana (Chair pose), Bhadrasana (Butterfly pose), Yastikasana (Stick pose), Pranayama such as bhramari (bee breath), ujjayi (ocean breath), nadi shodhana (sweet breath), Dirgha (three part breath).

\section{Asana}

Utkatasana: The patients were asked to stand erect with feet 12 inches apart, keeping their feet parallel to each other. Patients were asked to Inhale for 2 seconds and raise their heels and arms at shoulder level, with palms facing down: and then exhale slowly. It strengthens thigh and pelvis muscles.

Bhadrasana: The patients were made to sit on the mat and their legs were fully stretched, keeping their legs in contact with the mat. The patients were instructed to sit in erect without leaning forward and then place their hands-on knees or thighs. The patients maintained this position for few seconds. Strengthens the inner thighs and pelvic region.

Yastikasana: The patients were made to lie in supine position. The patients were instructed to keep their body in straight with feet together. Hands rest on the sides. The patients were asked to inhale and raise their hands, rest of them placing on the floor and then 
stretch upward by pushing their toes. And then slowly exhale and come back to the normal. The patient repeated this position for 3-4 times. This asana was helpful to correct posture and body get stretch, relieves the body tension.

\section{Pranayama}

Bhramari: This is also called as Bee breath technique which involves breathing in slowly through both nostrils. While exhaling the subject must use the throat to make a soft sound like the buzzing of bee.

Ujjayi: Also called as Ocean breath. This requires intake of air slowly through nose, filling lung entirely and exhaling completely until the abdomen empties out and compresses a bit while breathe in and out the subject needs to create a soft steady hissing sound.

Nadi Shodhana: otherwise called as Sweet breath technique. Nadi in Sanskrit means flow and shodhana means to cleanse. It is simple technique to follow and focuses on breathing through alternate nostrils.

Dirgha: Three part breath or complete breath. It involves three stages of breathing- high breathing, mid breathing- low breathing.

\section{Data analysis}

The data collected tabulated and analyzed using both descriptive and inferential statistics. statistical package for social science (SPSS) version 24 were used to assess the parameters. Paired t-test was adopted to find the statistical difference within the groups and independent t-test (Student's t-test) was adopted to find the statistical difference between the groups.

\section{RESULTS}

On comparing the mean values of group, A and group B on centre for epidemiological studies depression scale score, it shows significant decrease in the post test mean values (group A - aerobics) shows (24.60) and (group B - yoga) (24.40) at P > 0.05 . Both the groups are equally effective in decreasing the depression and maternal anxiety among primiparous women. Hence null hypothesis is accepted. On comparing pre-test and post-test within group A and group B on centre for epidemiological studies depression scale score shows highly significant difference in mean values at $\mathrm{P} \leq 0.001$.

Table 1: Comparison of centre for epidemiological studies -dépressions scale score between group A and group B in pre and post test

\begin{tabular}{|c|c|c|c|c|c|c|c|}
\hline \multirow{2}{*}{ \#CFESD } & \multicolumn{2}{|c|}{ \#Group A } & \multicolumn{2}{|c|}{ \#Group B } & \multirow{2}{*}{ T Test } & \multirow{2}{*}{ df } & \multirow{2}{*}{ Significance } \\
\cline { 2 - 7 } & Mean & S.D. & Mean & S.D. & & \\
\hline Pre-test & 46.66 & 5.06 & 45.66 & 5.05 & 0.541 & 28 & $0.593^{*}$ \\
\hline Post test & 24.60 & 3.81 & 24.40 & 3.77 & 0.144 & 28 & $0.886^{*}$ \\
\hline
\end{tabular}

\#Group A: Aerobic, \#Group B: Yoga. (*- P > 0.05)

The above table reveals the Mean, Standard Deviation (S.D), t-test, degree of freedom(df) and pvalue of the Centre for Epidemiological Studies Depression Scale between (group A) \& (group B) in pretest and post-test.

This table shows that there is no significant difference in pretest \& post-test values of the centre for epidemiological studies depression scale between group A pre-test (46.66) post-test (24.60) and group $\mathrm{B}$ pre-test (45.66) post-test $(24.40) .(* \mathrm{P}>0.05)$ (Graph-I).

Both the groups show significant decrease in the post test means group-A (24.60) and group-B (24.40) are equally effective in decreasing the depression and maternal anxiety.

Table 2: Comparison of centre for epidemiological studies depression scale score within group A and group B between pre and post-test values

\begin{tabular}{|l|c|c|c|c|c|c|}
\hline \multirow{2}{*}{ \#CFESD } & \multicolumn{2}{|c|}{ Pre-test } & \multicolumn{2}{c|}{ Post-test } & \multirow{2}{*}{ T Test } & \multirow{2}{*}{ Significance } \\
\cline { 2 - 6 } & Mean & S.D. & Mean & S.D. & & \\
\hline Group A & 46.66 & 5.06 & 24.60 & 5.05 & 18.87 & $0.000^{* * * *}$ \\
\hline Group B & 45.66 & 5.05 & 24.40 & 3.77 & 17.42 & $0.000^{* * *}$ \\
\hline
\end{tabular}

\#Group A: Aerobic, \#Group B: Yoga. (***- P $\leq 0.001)$

The above table reveals the mean, standard deviation (S.D), t-value and $\mathrm{p}$-value of the centre for epidemiological studies depression scale score between pre-test and post-test within group A and group B. Based on the centre for epidemiological studies depression scale score, it shows that there is a statistically highly significant difference between the pretest and post-test values within group $\mathrm{A}$ and group $\mathrm{B}(* * *-\mathrm{P} \leq 0.001)$.

\section{DISCUSSION}

The present study to compare the effectiveness of an aerobic exercise and yoga on depression and maternal anxiety orienting among primiparous women, Anxiety and depression are developed at the time of pregnancy. It occurs more frequently during the II and III trimesters. Around 40\% -70\% of women suffer from depression and anxiety and it most commonly occurs in the primiparous women 
due to worry about the upcoming labor, fear of childbirth. Physiologically the depression and anxiety occur by high cortisol level which is secreted by action of sympathetic nervous system. Some women manage with support of medications, pharmacological treatment such as anti-anxiety and anti-depressant drugs were effective in reducing the severity of depression and anxiety, but has side effects such as birth defects, premature birth, constipation, nausea, vomiting, etc., however the aerobic exercise and yoga shows satisfactory results to decrease the severity of depression and anxiety. Field et al., 2013 concluded that both yoga and social support were found to be effective in reducing depression, anxiety, anger in pregnancy and also during postpartum follow up (9). Shohani et al., 2018 concluded that yoga has an effective in role in reducing stress anxiety and depression (10). Arizabaleta et al., 2010 the study concluded that a supervised 3 month program of primarily Aerobic exercise during the pregnancy helps to improve health related quality of life (11). El-Rafiel et al., proposed that exercise during pregnancy was positively associated with reduce depression (12). In our present study we compared the effectiveness of aerobic exercise and yoga on depression and maternal anxiety on primiparous women. The study included second and third trimester women and excluded heart disease, lung disease, placenta abnormality, history of vaginal bleeding in second and third trimester, hypertension and several neurological diseases. Group A received aerobic exercise such as low step level, stationary bicycling, treadmill walking. The treatment session consisted of warm up for 10 minutes, aerobic exercise for 30 minutes. Group B received asana (utkatasana, bhadrasana, yastikasana) and pranayama (bhramari, ujjiayi, nadi shodhana, dirgha). Centre for epidemiological studies depression scale (CES-D), was used to analyses the symptoms of depression and anxiety, and it consists of 20 questions. The result of the study found that both the Groups are equal. When comparing both the groups there was significant decrease in the severity of depression and anxiety in both the groups the mean values of CES $-\mathrm{D}$ scale score in group A pre-test (46.66) and post-test (24.60) and group B pretest (45.66) and post- test (24.40). According to the result of this study aerobic exercise and yoga both significantly decrease the severity of depression and anxiety.

\section{CONCLUSION}

This study concludes that the aerobic exercise and yoga had equal effects with primiparous pregnant women suffering from depression and maternal anxiety. Both aerobic exercise and yoga can be considered as the better choice of treatment to reduce depression and anxiety than pharmacological treatment.

\section{CONFLICT OF INTEREST}

Authors declare that there is no conflict of interest.

\section{REFERENCES}

1. Sabari, Y., Nabel, H. The Impact of anxiety and depression during pregnancy on fetal growth and the birth outcome. Egyptian Journal of Psychiatry. 2015; 36(2): 95-100.

2. Arora, P., Aeri, B. T. Burden of antenatal depression and its risk factor in Indian settings: A systematic review. Indian Journal of Medical Specialties. 2019; 10: 55-60. DOI: 10.4103/INJMS.INJMS_36_18

3. Gong, H., Ni, C., Shen, X., Wu, T., Jiang, C. Yoga for prenatal depression: A systemic review and meta-analysis. BMC Psychiatry. 2015; 15: 14.

4. Field, T. Prenatal anxiety effects: A review. Infant Behavior \& Development. 2017; 49: 120-128.

5. Pillay, P. S., Piercy, C. N., Tolppanen, H., Mebazaa, A. Physiological changes in pregnancy. Cardiovascular Journal of Africa. 2016; 27(2): 89-94.

6. Prather, H., Spitznagle, T. M., Hunt, D. Benefits of exercise during pregnancy. Physical Medicine \& Rehabilitation. 2012; 4(11): 845-850.

7. Curtis, K., Weinrib, A., Katz, J. Systemic review of yoga for pregnant women: current status and future directions. 2012 Article ID 715942 | https://doi.org/10.1155/2012/715942

8. William, W. E., Corey, S., Michele, Y., Carles, M., Allen, T. Center for epidemiologic studies depression scale: Review and revision (CESD and CESD-R). 2004; In Maruish, Mark E. (ed.). The use of psychological testing for treatment planning and outcomes assessment: Instruments for adults. 3 (3rd ed.). Mahwah, NJ: Lawrence Erlbaum Associates. pp. 363-377. ISBN 978-0-8058-4331-6. OCLC 55130556.

9. Field, T., Diego, M., Delgado, J., Medina, L. Yoga and social support reduce prenatal depression, anxiety and cortisol. Journal of bodywork and movement therapies. Journal of Bodyworks and Movement Therapies. 2013 Oct; 17 (4): 397-403.

10. Shohani, M., Badfar, G., Nasirkandy, M. P., Kaikhavani, S., Rahmati, S., Modmeli, Y., et al., The effect of yoga on stress, anxiety and depression in women. International Journal of Preventive Medicine. 2018; 9:21.

11. Arizabaleta, A. V. M., Buitrago, L. O., de Plata A. C. A., Escudero, M. M., Ramirez-Velez, R. Aerobic exercise during pregnancy improves health- related quality of life: a randomized trial. Journal of Physiotherapy. 2010; 56(4): 253-258.

12. El-Rafie, M., Khafagy, G., Gamal, M. Effect of aerobic exercise during pregnancy on antenatal depression. Department of Public Health. 2016; 2016: 53-57. DOI https://doi.org/10.2147/IJWH.S94112 